\title{
Impacto socioeconómico del Programa Pago por Servicios Ambientales, modalidad reforestación, en el noroeste de Costa Rica*
}

\section{Socio-economic Impact of the Payment for Environmental Services Program, Reforestation Mode, in Northwest Costa Rica}

\section{Carla Padilla Salas**, Sergio A. Molina-Murillo***}

Recibido: 2016-07-28 // Aprobado: 2016-08-30// Disponible en linea: 2017-01-30

Cómo citar este artículo: Padilla Salas, C. y Molina-Murillo, S. A. (2017). Impacto socioeconómico del Programa Pago por Servicios Ambientales, modalidad reforestación, en el noroeste de Costa Rica. Ambiente y Desarrollo, 21(40), 73-91. https://doi.org/10.11144/Javeriana.ayd21-40.isps doi:10.11144/Javeriana.ayd21-40.isps

\section{Resumen}

El objetivo de este estudio fue evaluar el impacto socioeconómico del Programa Pago por Servicios Ambientales (PPSA) en beneficiarios que gestionaron contratos bajo la modalidad reforestación entre 1998 y el 2013, mediante dos organizaciones de base forestal (OBF) en el noroeste de Costa Rica. En primer lugar, se identificaron las características socioeconómicas de los beneficiarios y luego se estableció, validó y aplicó un set de principios, criterios e indicadores para evaluar el impacto del PPSA en las dimensiones social y económica. En general, los resultados indican que el programa ha fortalecido las capacidades locales para el desarrollo forestal de la zona, principalmente por medio de la participación activa de las OBF y los beneficiarios, aunque la percepción de dichos beneficios pareciera disminuir en el tiempo.

Palabras clave: bienestar social; método de evaluación; organizaciones de base comunitaria; reforestación; sostenibilidad

\footnotetext{
* $\quad$ Este artículo es resultado del proyecto de investigación Impacto socioeconómico del Programa Pago por Servicios Ambientales, modalidad reforestación, en el noroeste de Costa Rica, realizado como tesis por Carla Padilla Salas bajo la tutoría del profesor Sergio A. Molina Murillo.

** Ingeniera forestal, licenciada en Ciencias Forestales con énfasis en Comercio de Productos Forestales, Universidad Nacional de Costa Rica. Correo electrónico: carla-ps@hotmail.com

*** Doctor en Economía Ambiental, académico e investigador de la Escuela Ciencias Ambientales de la Universidad Nacional de Costa Rica. Investigador en la Unidad de Recursos Forestales, Universidad de Costa Rica. Correo electrónico: sergiomolina@una.cr
} 


\begin{abstract}
The purpose of this study was to evaluate the socioeconomic impact of the Payment for Environmental Services Program (PPSA) on beneficiaries who managed reforestation-mode contracts between 1998 and 2013 through two forest-based organizations (OBF) in northwest Costa Rica. First, we identified the socioeconomic characteristics of the beneficiaries and established, validated, and applied a set of principles, criteria, and indicators to assess the impact of the PPSA on the social and economic dimensions. Overall, results indicate that the program has strengthened local capabilities for forest development in the area, mainly through the active involvement of the OBFs and beneficiaries, although the perception of these benefits appears to decrease over time.
\end{abstract}

Keywords: social welfare; evaluation method; community-based organizations; reforestation; sustainability 


\section{Introducción}

La consolidación del mecanismo de pago por servicios ambientales —o ecosistémicos- en Costa Rica se ha gestado organizacional e institucionalmente en las últimas cinco décadas, a través de un proceso de innovación ambiental, social, económico, político y cultural. El Programa de Pago por Servicios Ambientales (PPSA) de Costa Rica es el primer programa nacional de esta naturaleza en un país en desarrollo (Pagiola, 2008). Oficialmente, nació en 1997 como un mecanismo financiero alternativo para compensar a propietarios por los servicios ambientales que proveen sus bosques naturales y plantaciones forestales y así detener una de las tasas de deforestación más altas del mundo, como era la que venía padeciendo el país. Su objetivo principal es suministrar los servicios ambientales de protección de la biodiversidad, mitigación del efecto invernadero, belleza escénica y protección del recurso hídrico (Ley Forestal N. ${ }^{\circ}$ 7575, artículo 3, 1997).

El PPSA costarricense ha sido reconocido internacionalmente (p. ej., Porras et al., 2012; Engel et al., 2008) como un instrumento de mercado que permite compensar estos servicios a través de distintas modalidades (p. ej., conservación de bosques, reforestación, manejo sostenible del bosque, regeneración natural y plantación de árboles en sistemas agroforestales). Actualmente el PPSA forma parte de la estrategia nacional de cambio climático y constituye un elemento central en la propuesta de desarrollo que se basa en la protección de los recursos naturales, el turismo sostenible y el desarrollo de mecanismos limpios de crecimiento (Camacho y Solano, 2010).

Además de los ecosistemas, se espera que el programa impacte directamente aspectos sociales y económicos de los participantes. Si bien su definición jurídica no le dicta el objetivo directo de reducir la pobreza, este sí se encuentra implícito al priorizar los pequeños y medianos productores forestales (Contraloría General de la República [CGR], 2011; Ortiz et al., 2003). No obstante, hoy se cuestiona el aumento en la calidad de vida de los beneficiarios por participar en programas de PSA (Daw et al., 2016; Arriagada et al., 2015; Ferraro et al., 2014).

Con el fin de evaluar y monitorear dichos impactos, se han desarrollado múltiples estrategias metodológicas. Entre ellas, por ejemplo, la medición directa de la cantidad o la calidad del agua; el modelado, que utiliza la relación entre las intervenciones propuestas y los resultados anticipados de los servicios ecosistémicos; o la construcción de sistemas de indicadores, que permite el uso de información primaria y secundaria en la identificación, selección y evaluación de impactos con precisión y fiabilidad de manera eficiente (Smith, 2013; FIDA, 2003). Esta última estrategia crea la opción de trabajar con mayor facilidad las dimensiones social y económica, o incluso enfoques como el de medios de vida sostenibles (MVS) que contempla diferentes tipos de capitales: humano, social, financiero, físico y natural (Muñoz, 2012; Miranda et al., 2003).

Sin embargo, para el PPSA costarricense hoy se carece de un análisis comprensivo, tal y como lo señala un informe de la Contraloría General de la República (CGR, 2011; p. 35). En dicho informe se seńala la necesidad de evaluar integralmente los impactos ambientales, económicos y sociales del programa, requisito para la rendición de cuentas y una adecuada medición de su efectividad en el tiempo. Específicamente, se menciona la ausencia de indicadores e información sistematizada que permita la medición del desempeño y la gestión del programa. Otros autores, como Porras et al. (2012), sostienen que para mejorar el éxito futuro del programa, se requiere diseñar con claridad un sistema de definición y evaluación de impactos sociales.

Si bien algunos estudios han tratado de hacer abordajes más inclusivos (Fondo Nacional Financiamiento Forestal [Fonafifo], 2015; Molina-Murillo et al., 2014), la mayoría ha medido la efectividad del programa con base en la participación (número de contratos) o en cambios de la cobertura boscosa, lo cual limita la evaluación de dichos programas (Borner et al., 2017; Daw et al., 2016; Engel et al., 2008). Valenciano et al. (2013) y Porras et al. (2012) por ejemplo, han recomendado evaluar el PPSA considerando la cantidad de empleos generados y el aumento en las capacidades para manejar las plantaciones o bosques. 
Adicionalmente y considerando que la experiencia del PPSA ha favorecido el desarrollo de una institucionalidad y distintas formas organizativas (Rosa et al., 2004; Camacho et al., 2000) claves en el éxito de programas de esta naturaleza (Borner et al., 2017; Huber-Stearns et al., 2013; Pham et al., 2010), se considera relevante investigar el desempeńo de las dimensiones social y económica, mediado por organizaciones forestales de base (OFB) que apoyan y acompañan en los procesos de promoción y seguimiento técnico del programa.

Por tanto, el objetivo de este estudio es evaluar el impacto socioeconómico del PPSA en los beneficiarios que gestionaron contratos bajo la modalidad de reforestación, mediante dos OBF, en el noroeste de Costa Rica entre los años 1998 y 2013. Después de una breve descripción del PPSA de Costa Rica, se explica el proceso metodológico utilizado para desarrollar, validar y aplicar el sistema de evaluación, seguido por los resultados obtenidos en cuanto al impacto en beneficiarios del PPSA en las dimensiones social y económica. Finalmente, después de la discusión se presentan las principales conclusiones.

\section{Breve descripción del funcionamiento del PPSA en su modalidad reforestación}

A escala nacional, la demanda de servicios ambientales es manejada por el Estado mediante el Ministerio de Ambiente y Energía (MINAE), el cual delega según la normativa vigente (Reglamento a la Ley Forestal N. ${ }^{\circ} 7575$, artículo 61-62, 1997), la ejecución al Fondo Nacional de Financiamiento Forestal (Fonafifo) en coordinación con el Sistema Nacional de Áreas de Conservación (Sinac). De acuerdo a lo estipulado, cada beneficiario recibe el PSA y acepta ceder a Fonafifo por un periodo igual a la vigencia del contrato, los derechos sobre los servicios ambientales generados en el área sujeta a contrato.

Aunque el programa se financia principalmente con el 3.5\% del impuesto único a los combustibles, se han gestionado apoyos a través de múltiples proyectos y negociaciones nacionales e internacionales (Rosa et al., 2004). Producto de este impuesto y préstamos de cooperación internacional, el Gobierno de Costa Rica financió cerca del 88\% de este programa hasta el año 2010 (Legrand et al., 2016).

\section{PPSA de Costa Rica}

\begin{tabular}{|c|c|}
\hline $\begin{array}{l}\text { Demanda nacional } \\
\text { GOBIERNO }\end{array}$ & $\begin{array}{c}\text { *Demanda internacional (carbón, } \\
\text { biodiversidad, agencias) }\end{array}$ \\
\hline $\begin{array}{c}\text { Intermediario principal } \\
\text { FONAFIFO }\end{array}$ & $\begin{array}{l}\text { Compra los "derechos" de servicios ambientales } \\
\text { (en "paquete") } \\
\text { "Desempaca" estos derechos y los vende a } \\
\text { diferentes usuarios } \\
\text {. Administra el programa a nivel nacional }\end{array}$ \\
\hline $\begin{array}{l}\text { Facilitadores } \\
\text { OBF y Regentes }\end{array}$ & $\begin{array}{l}\text { Información } \\
\text { Apoyo técnico } \\
\text { Cobran hasta } 18 \% \text { del pago }\end{array}$ \\
\hline $\begin{array}{c}\text { Finqueros } \\
\text { (Proveedores de } \\
\text { servicios ambiantales) }\end{array}$ & $\begin{array}{l}\text { Privados } \\
\text { Reciben un pago por el "paquete" de servivios } \\
\text { ambientales ( } \phi \text { /ha/año; ф/árb) } \\
\text { Contratos 5-16 años }\end{array}$ \\
\hline
\end{tabular}

Figura 1. Esquema de funcionamiento del PPSA en Costa Rica

Fuente: modificado de Porras et al. (2012) 
Los interesados en ingresar al programa pueden solicitarlo de forma individual con un regente forestal o a través de proyectos regentados por OBF, quienes apoyan técnicamente cobrando distintos porcentajes de honorarios (Ministerio de Ambiente, Energía y Telecomunicaciones [Minaet], 2009; Zbinden y Lee, 2004). Pueden participar personas físicas o jurídicas, propietarios, arrendatarios y usufructuarios de inmuebles inscritos en el Registro Nacional de Costa Rica (figura 1).

Los contratos de reforestación se pagan durante los primeros cinco ańos; en el primer año se paga el $50 \%, 20 \%$ en el segundo año, y así sucesivamente, un 15, 10 y 5\%, hasta llegar al quinto año. Existe además la posibilidad de que el primer tracto sea por adelantado, si el área máxima es de 50 ha. El área mínima en proyectos de reforestación es de 1 ha y el máximo de 300 ha. Su monto era diferenciado, dependiendo de si las especies sembradas eran nativas o no, y sus áreas prioritarias eran definidas según el potencial productivo, el nivel de vinculación bosque-industria y aquellos que utilizaran material genético modificado o semillas certificadas. A partir del año 2015 y bajo el Decreto Ejecutivo N. ${ }^{\circ}$ 39083-MINAE, se presentan cambios significativos, con aumentos en los montos diferenciados según especies de rápido o mediano crecimiento, condicionado a una mayor exigencia en los rendimientos de la producción, de acuerdo a lo señalado teóricamente por Wunder $(2005,2015)$.

Para priorizar la asignación de los insuficientes pagos entre los múltiples solicitantes, el PPSA ha desarrollado criterios que pretenden maximizar tanto el abastecimiento de servicios ambientales como los beneficios socioeconómicos en las comunidades locales. Por ejemplo, se priorizan tierras aledañas a las áreas protegidas o zonas de recarga hídrica en la dimensión ambiental, o el índice de desarrollo social y el tamaño de finca como criterios socioeconómicos (Minae, 2015). Aunque estos criterios de priorización podrían usarse para evaluar el desempeño del programa, el asunto es complejo y la evidencia no es contundente sobre los efectos que los pagos tienen en la calidad de vida de los participantes y sus familias (Porras et al., 2012; CGR, 2011; Ortiz et al., 2003). Esta complejidad es evidenciada por estudios recientes (Robalino et al., 2014; Alpízar et al., 2013; Muñoz, 2012), los cuales sugieren que el impacto socioeconómico de los pagos es mayor en sitios con altas pendientes, donde los costos de oportunidad no son tan altos comparados con sitios planos.

Las OFB, en la forma de cooperativas agrícolas, fundaciones, centros agrícolas cantonales, asociaciones y federaciones campesinas apoyan los trámites de PSA y acompañan al Estado en la promoción y el seguimiento técnico del programa. Entre sus principales funciones se encuentran: venta de semillas certificadas y material genético mejorado, servicio de regencia forestal y asesoría técnica a las distintas modalidades del PSA, y asesoría en comercialización de madera, entre otras. Cada año se asignan cuotas a las OFB para la colocación de los PSA, y, específicamente, en la modalidad de reforestación estas aportaron el $25 \%$ del área total tramitada a escala nacional durante el periodo 2010-2014, con un promedio de finca de 16.15 ha, lo cual evidencia el apoyo a pequeńos y medianos productores (Chavarría, 2014). El $80 \%$ de la inversión se dirige a la contratación de personas -14 500 empleos directos en el año 2014-, lo cual dinamiza el desarrollo local (Oficina Nacional Forestal [ONF], 2012, 2014; Méndez y Salazar, 2010; Arias, 2004).

\section{Metodología}

El estudio se realizó en la provincia de Guanacaste, en el Pacífico norte de Costa Rica (figura 2). Esta es la región del país con el mayor área reforestada con fines comerciales, principalmente por las especies de teca (Tectona grandis) y melina (Gmelina arborea), con 20307.8 y 2447.1 ha, respectivamente (Instituto Nacional de Estadísticas y Censos [INEC], 2015). En esta región se encuentran los centros agrícolas de Hojancha $(\mathrm{CACH})$ y Nandayure $(\mathrm{CACN})$, OBF posicionadas entre las primeras cinco a escala nacional en términos de mayor cantidad de área en contratos PSA de reforestación durante el periodo 2000-2014 (Chavarría, comunicación personal, 29 de abril, 2016). Aunque en otras zonas del país se han realizado estudios del PPSA con algún componente social (Molina-Murillo et al., 2014; Alpízar et al., 2013; Arraigada et al., 2010; Barton et al., 2009; Sierra y Russman, 2006; 
Zbind y Lee 2005; Ortiz et al., 2003; Miranda et al., 2003), ninguno se ha hecho específicamente para la modalidad de reforestación, ni tampoco en esta zona de estudio, por lo que cobra importancia la realización de un estudio de caso como este, donde se aborda metodológicamente un fenómeno en su entorno real, lo cual cuenta con una gran utilidad, pues se trata de un método valioso en la medición y el registro de la conducta de las personas que se ven involucradas, así como del conocimiento de su percepción, la cual, al fin y al cabo, es la que determina su participación en el programa y su éxito o fracaso (Martínez, 2006).

La población en estudio se constituyó de beneficiarios del PPSA que gestionaron sus contratos de reforestación en fincas ubicadas en el cantón de Hojancha, por medio del CACH, y en Nandayure con el CACN, durante los periodos: 1998-2000, 2005-2007 y 2011-2013. Estos periodos fueron elegidos con el propósito de tener representatividad de beneficiarios en las diferentes etapas del proceso de reforestación (p. ej., siembra y crecimiento inicial, etapa media que conlleva aplicación de raleos, turno final). El análisis se realizó con base en una población inicial de 113 beneficiarios, correspondientes a 80 contratos, con información facilitada por ambas OBF: Sinac y Fonafifo. Del total se pudieron clasificar 83 personas como beneficiarios localizables, a partir de los cuales se entrevistó una muestra de 41 beneficiarios ( 21 con el CACH y 20 con el CACN). Estos fueron seleccionados al azar y estratificados de manera proporcional al número de contratos por organización y periodo, con un error máximo de muestreo de $10.04 \%$.

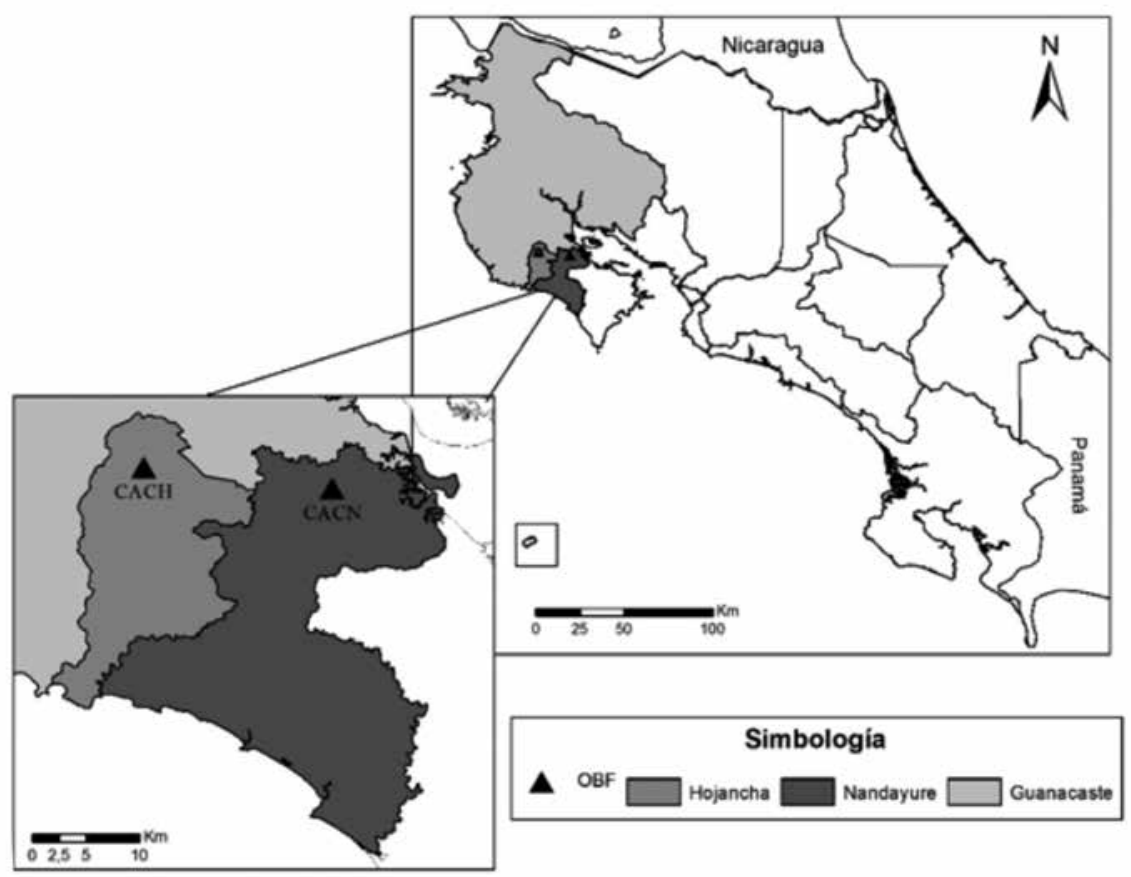

Figura 2. Mapa de ubicación del área de estudio 
En la primera fase se generó un perfil socioeconómico del productor forestal que ingresa al programa en esta modalidad y por medio de estas organizaciones. La información fue levantada mediante una entrevista semiestructurada diseñada para su aplicación en campo. A tal efecto, se elaboró un cuestionario con 61 preguntas para levantar información de variables sociodemográficas, la finca y generales del PSA; y otras para conocer, a través de escalas tipo Likert, el grado de satisfacción o mejora percibido por el beneficiario en determinado indicador (Muñoz, 2012). Con el fin de profundizar en el análisis del contexto sociocultural, se consideró el porcentaje de mujeres productoras, productores según grupo de edad y nivel de instrucción, así como otros datos colectados por el INEC durante el Censo Nacional 2011 y el Censo Agropecuario 2014.

En la segunda fase se diseñó un sistema de evaluación basado en principios, criterios e indicadores para medir el impacto del PPSA en la región de estudio (se muestran detalladamente en la tabla 2). Para esto, se construyó un esquema preliminar, considerando estudios nacionales e internacionales en el tema (p. ej. Molina-Murillo et al., 2014; Alpízar et al., 2013; Muñoz, 2012; Arraigada et al., 2010; Barton et al., 2009; Sierra y Russman, 2006; Zbind y Lee 2005; Ortiz et al., 2003; Miranda et al., 2003). Los indicadores considerados debían cumplir con ser medibles, válidos y con información accesible. La lista de principios, criterios e indicadores preliminares fue validada mediante una consulta a once expertos, entre ellos actores clave, regentes forestales y funcionarios de instituciones y organizaciones vinculadas (tabla 1). Por medio de la aplicación de un formulario en la plataforma Survey Monkey y siguiendo la técnica de análisis multicriterio (rateo), descrita por Morán et al. (2006) y McGinley y Finegan (2001), los expertos asignaron un peso entre 1 y 10, según la importancia dada a cada una de las dimensiones y los criterios que las componen. El valor de los criterios se dividió entre el número de los indicadores, por lo cual en caso de no poder ser medido o no estar disponible la información de un indicador, el valor total del criterio no se afecta (Molina-Murillo et al., 2014).

Tabla 1. Lista de expertos consultados en la validación de los principios, criterios e indicadores

\begin{tabular}{lll}
\hline \multicolumn{1}{c}{ Nombre } & \multicolumn{1}{c}{ Organización } & \multicolumn{1}{c}{ Cargo } \\
\hline Ina Porras & IIED-Reino Unido & Economista investigadora \\
\hline Olman Morales Soto & Aclac-Sinac-Minae & Educador ambiental \\
\hline Sonia Lobo Valverde & Sinac-minae & $\begin{array}{l}\text { Profesional en Gerencia } \\
\text { de Conocimiento y Uso }\end{array}$ \\
\hline Juan Robalino & Catie & Investigador asociado \\
\hline Gerardo Barrantes & $\begin{array}{l}\text { Instituto de Políticas } \\
\text { de Sostenibilidad }\end{array}$ & Director \\
\hline Bastiaan Louman & Catie & $\begin{array}{l}\text { Líder Cambio Climático y Cuencas } \\
\text { Hidrográficas }\end{array}$ \\
\hline Jhonny Méndez Gamboa & Codeforsa & Director ejecutivo \\
\hline Ronnie de Camino Velozo & Catie & $\begin{array}{l}\text { Director, Cátedra de Gestión } \\
\text { Forestal Territorial }\end{array}$ \\
\hline Emel Rodríguez Paniagua & ACT- Sinac- Minae & $\begin{array}{l}\text { Coordinador regional del Programa } \\
\text { de Desarrollo Forestal }\end{array}$ \\
\hline José Joaquín Rodríguez & Exfuncionario del CACH & Ingeniero forestal \\
\hline Luis Felipe Torres & CACN & Gerente \\
\hline
\end{tabular}


El trabajo con expertos generó un primer filtro y determinó la practicidad de utilizar los indicadores sugeridos (Morán et al., 2006). Según el resultado de este proceso de consulta y las observaciones de los expertos, se construyó una matriz evaluadora compuesta por cada principio, criterio e indicador y su respectivo puntaje, la cual a su vez permitió confeccionar el cuestionario que se aplicó durante los meses de noviembre del 2015 y enero del 2016 a los beneficiarios seleccionados. La mayoría de preguntas se orientó a medir la percepción de los participantes y evaluar los impactos (Muñoz, 2012).

En la última fase se sistematizaron y construyeron escalas de evaluación para los indicadores económicos 1.1, 1.3, 2.1 y 3.1, los cuales requirieron una categorización con el fin de establecer parámetros y asignar puntajes, al referirse al grado de satisfacción o mejora apreciado por el productor. Finalmente, el puntaje obtenido por cada principio, criterio e indicador se ingresó en la matriz evaluadora para así comparar la calificación final alcanzada con la calificación de referencia propuesta por los expertos. El análisis de los resultados conllevó la caracterización de los impactos socioeconómicos, considerando los puntos de vista de los beneficiarios, analizando la tendencia de las respuestas y utilizando información secundaria para ayudar a comprender las realidades expuestas por los participantes (Garay et al., 2004).

\section{Resultados y discusión}

\section{Perfil socioeconómico de los beneficiarios}

En Costa Rica los pagos recibidos por servicios ambientales han sido desde sus inicios gestionados, principalmente, por hombres. Entre los 1997 y 2014 se realizaron en total 14713 contratos en todas las modalidades, de los cuales únicamente 2207 (aproximadamente el 15\%) fueron a mujeres. En esta investigación, el $83 \%$ corresponde a contratos dirigidos por hombres. Esta realidad responde a una brecha en la posesión de propiedades entre hombres y mujeres, característica en toda Latinoamérica (León, 2011). El manejo desigual de la tierra se demuestra también en los datos recopilados por el INEC (2015) durante el censo agropecuario, los cuales indican que en ambos cantones el porcentaje promedio de mujeres productoras es únicamente de 12,14\%. En nuestro estudio se evidencia que incluso las poseedoras de contratos con frecuencia ignoran su estado, así como el de las correspondientes plantaciones forestales.

Con respecto a la variable edad, los proyectos de reforestación en la zona están siendo ejecutados mayormente por personas entre cuarenta y setenta años, con un promedio 55 años. Esta situación expone el desinterés por parte de generaciones más jóvenes de incursionar o continuar con las actividades de reforestación, lo cual pone en riesgo su sostenibilidad. Lamentablemente, en la zona el número de personas productoras agrícolas / forestales menores de cuarenta años va en descenso (INEC, 2011, 2015), con un sector primario cada vez más pequeño (33.3\% actualmente, comparado con $80 \%$ en el 2001) (Morales, 2016; Instituto Espańol de Comercio Exterior [ICEX], 2012; INEC, 2011; Instituto Costarricense de Estudios Sociales [ICES], 2001).

Entre los participantes predomina una escolaridad básica (primaria completa) y los oficios más frecuentes son: productor agropecuario y funcionario de instituciones relacionadas con el sector ambiental del país. Llamativamente, solo tres personas se dedican en forma exclusiva a la actividad forestal. El total de beneficiarios consultados no ha cambiado su domicilio en los últimos cinco años, y el $70 \%$ de ellos vive en la finca o en el mismo distrito de la propiedad bajo contrato PSA, lo cual potencializa el impacto generado sobre la economía local (Miranda et al., 2003). 
Sobresale la participación de pequeńos y medianos productores, con promedios de 52.72 ha para la propiedad y de 7.83 ha para el área bajo PSA. Serrano (2005) indica que en Hojancha la tenencia de la tierra es predominantemente privada (74\%), aspecto que facilita el acceso a financiamiento y crédito. No obstante, los dueños de propiedades más grandes colaboran de manera indirecta con los productores más pequeños, ya que estos manifestaron gestionar sus contratos con la $\mathrm{OBF}$, a fin de apoyar a la organización local.

El $44 \%$ de los beneficiarios cuenta con contratos de reforestación adicionales al considerado en este estudio, con un tamańo promedio de proyecto de 8.35 ha, lo cual indica que la actividad forestal es llevada a cabo por muchos productores que plantan pequeñas áreas de tierra. El PPSA también es utilizado para conservar y proteger el bosque, con un tamaño promedio de 62.33 ha, y esto es sumamente importante si se considera la crisis ganadera que en los años setenta generó pobreza, deforestación y degradación de suelos y fuentes de agua, por lo cual las personas comenzaron a incorporar enfoques más integrales y diversificados de producción en terrenos donde coexisten bosques, ganadería y cultivos, y de tal manera se fortaleció el desarrollo forestal de la zona (Madrigal et al., 2012; Aguilar e Iza, 2009).

Por lo general, la actividad forestal es financiada con los desembolsos del PSA y recursos propios. Del total de entrevistados, solamente el $5 \%$ solicitó algún tipo de crédito. El desconocimiento o los costos de transacción podrían ser las razones, aunque entre los productores consultados pareciera destacar su cultura por la actividad forestal. El $56 \%$ aumentaría la cantidad de hectáreas reforestadas y, aun sin un pago de por medio, el $70 \%$ seguiría dedicándose a la actividad, motivado por los ingresos de la venta de madera, la generación de empleo, una menor inversión comparada con otros cultivos y la utilización de terrenos con pendientes pronunciadas.

\section{Evaluación del impacto del Programa Pago por Servicios Ambientales para todos los contratos}

En Costa Rica el PPSA se creó con el objetivo de detener la deforestación sufrida desde la década de los sesenta y asegurar la provisión de servicios ecosistémicos esenciales; sin embargo, estudios han comprobado que este programa también genera impactos en la condición socioeconómica de sus beneficiarios (Molina-Murillo et al., 2014; Alpízar et al., 2013; Camacho et al., 2010; Barton et al., 2009; Garay et al., 2004; Miranda et al., 2003). A través de un análisis general del programa en sus primeros quince años, Porras et al. (2012) sostienen que, para mejorar su éxito futuro, se requiere diseñar con claridad un sistema de definición y evaluación de impactos sociales. A partir de tal aserto, se realiza el presente estudio, donde utilizamos una matriz constituida por dos principios, doce criterios y dieciséis indicadores (tabla 2). Los expertos asignaron un peso de $50.3 \%$ a la dimensión social y de $49.7 \%$ a la dimensión económica. El desempeño, evaluado con los beneficiarios, resultó en $33.63 \%$ para la dimensión social y $31.73 \%$ para la económica.

Los criterios sociales considerados más importantes para los expertos fueron el 3 y el 4, los cuales evalúan el fortalecimiento de las capacidades del productor a través de capacitaciones, así como la participación activa del beneficiario en los distintos procesos productivos de su plantación forestal. Ambos recibieron un puntaje de $15.16 \%$. El criterio social con menor puntaje asignado, con un $12.64 \%$, fue el número 6 , el cual se relaciona con la mejoría en la relación que tienen los beneficiarios con las distintas instituciones de apoyo. Por su parte, el criterio económico 3, que mide los impactos sobre la generación de empleo familiar, fue el más importante, con un $22.63 \%$, mientras que el considerado menos importante fue el 2, relacionado con el aporte del PSA durante el establecimiento y el mantenimiento de la plantación. En la figura 3 se detallan tanto el valor de importancia asignado por los expertos como el valor obtenido durante la evaluación en el sitio de estudio. 
Tabla 2. Matriz evaluadora del impacto socioeconómico del Programa Pago por Servicios Ambientales y los respectivos pesos asignados por expertos

\begin{tabular}{|c|c|c|c|}
\hline \multicolumn{4}{|l|}{ Dimensión social (Peso: $50.3 \%$ ) } \\
\hline \multicolumn{4}{|c|}{$\begin{array}{l}\text { Principio: el Programa de Pago por Servicios Ambientales es un instrumento financiero que mejora } \\
\text { las condiciones sociales de los beneficiarios. }\end{array}$} \\
\hline Criterio & $\begin{array}{l}\text { Peso del } \\
\text { criterio }\end{array}$ & Indicador & $\begin{array}{l}\text { Peso del } \\
\text { indicador }\end{array}$ \\
\hline $\begin{array}{l}\text { 1. Apoyo del PPSA a pequeños } \\
\text { propietarios }\end{array}$ & 14.08 & $\begin{array}{l}\text { 1.1. Cantidad de beneficiarios con propieda- } \\
\text { des iguales o menores a } 50 \text { ha con respecto } \\
\text { al total encuestado }\end{array}$ & 14.08 \\
\hline $\begin{array}{l}\text { 2. Mecanismos de gestión par- } \\
\text { ticipativa en los beneficiarios } \\
\text { del PSA }\end{array}$ & 13.72 & $\begin{array}{l}\text { 2.1. Cantidad de beneficiarios que se incor- } \\
\text { poraron a organizaciones a partir de su par- } \\
\text { ticipación en el PPSA con respecto al total } \\
\text { encuestado }\end{array}$ & 13.72 \\
\hline \multirow[t]{2}{*}{$\begin{array}{l}\text { 3. Fortalecimiento de capaci- } \\
\text { dades mediante el acceso a la } \\
\text { capacitación }\end{array}$} & \multirow[t]{2}{*}{15.16} & $\begin{array}{l}\text { 3.1. Cantidad de beneficiarios que acudieron } \\
\text { a actividades de formación (talleres, reunio- } \\
\text { nes, días de campo, capacitaciones), coor- } \\
\text { dinadas por Fonafifo, ONF, el respectivo } \\
\text { Centro Agrícola o alguna otra organización } \\
\text { relacionada con el PPSA, con respecto al } \\
\text { total encuestado }\end{array}$ & 7.58 \\
\hline & & $\begin{array}{l}\text { 3.2. Nivel de mejora percibida por el benefi- } \\
\text { ciario en la capacidad técnica para el manejo } \\
\text { de las plantaciones forestales. }\end{array}$ & 7.58 \\
\hline $\begin{array}{l}\text { 4. Participación activa del be- } \\
\text { neficiario dentro de la planta- } \\
\text { ción forestal bajo PSA }\end{array}$ & 15.16 & $\begin{array}{l}\text { 4.1. Cantidad de beneficiarios que participan } \\
\text { activamente en el diseño, establecimiento y } \\
\text { manejo de la plantación forestal bajo PSA, } \\
\text { con respecto al total encuestado. }\end{array}$ & 15.16 \\
\hline \multirow{2}{*}{$\begin{array}{l}\text { 5. Beneficios de la producción } \\
\text { de bienes y servicios de la plan- } \\
\text { tación forestal bajo PSA }\end{array}$} & \multirow[t]{2}{*}{14.44} & $\begin{array}{l}5.1 \text { Nivel de mejora percibida por el beneficia- } \\
\text { rio en su nivel de conciencia sobre los servi- } \\
\text { cios ambientales que brindan las plantaciones } \\
\text { forestales. }\end{array}$ & 7.22 \\
\hline & & $\begin{array}{l}\text { 5.2. Nivel de satisfacción del beneficiario con } \\
\text { los resultados que obtiene respecto a los bienes } \\
\text { y servicios de la plantación forestal bajo PSA. }\end{array}$ & 7.22 \\
\hline $\begin{array}{l}\text { 6. Relación con instituciones } \\
\text { de apoyo }\end{array}$ & 12.64 & $\begin{array}{l}\text { 6.1. Nivel de mejora considerada por los be- } \\
\text { neficiarios en su relación con Fonafifo y el } \\
\text { respectivo centro agrícola desde su partici- } \\
\text { pación en el PPSA }\end{array}$ & 12.64 \\
\hline $\begin{array}{l}\text { 7. Legalización de la tenencia } \\
\text { de la tierra }\end{array}$ & 14.80 & $\begin{array}{l}\text { 7.1 Cantidad de beneficiarios que actuali- } \\
\text { zan y ordenan la documentación relativa a } \\
\text { su propiedad debido a la participación en el } \\
\text { PPSA, con respecto al total encuestado }\end{array}$ & 14.80 \\
\hline \multicolumn{4}{|c|}{ Dimensión económica (peso: 49.7\%) } \\
\hline \multicolumn{4}{|c|}{$\begin{array}{l}\text { Principio: el Programa de Pago por Servicios Ambientales es un instrumento financiero que contri- } \\
\text { buye al bienestar económico de los beneficiarios. }\end{array}$} \\
\hline
\end{tabular}




\begin{tabular}{|c|c|c|c|}
\hline Criterio & $\begin{array}{l}\text { Peso del } \\
\text { criterio }\end{array}$ & Indicador & $\begin{array}{l}\text { Peso del } \\
\text { indicador }\end{array}$ \\
\hline \multirow{3}{*}{$\begin{array}{l}\text { 1. Impacto de los ingresos per- } \\
\text { cibidos por PSA sobre la eco- } \\
\text { nomía del beneficiario }\end{array}$} & \multirow{3}{*}{21.25} & $\begin{array}{l}\text { 1.1. Porcentaje que representan los ingresos } \\
\text { del PSA sobre la economía del beneficiario } \\
\text { con respecto a otros ingresos. }\end{array}$ & 7.08 \\
\hline & & $\begin{array}{l}\text { 1.2. Mejora percibida en la economía del } \\
\text { beneficiario debido a ingresos netos prove- } \\
\text { nientes del PSA }\end{array}$ & 7.08 \\
\hline & & $\begin{array}{l}\text { 1.3. Porcentaje de inversión del ingreso PSA } \\
\text { en aspectos como: gastos del hogar, salud, } \\
\text { educación, entre otros }\end{array}$ & 7.08 \\
\hline $\begin{array}{l}\text { 2. Aporte neto del PSA a los } \\
\text { costos por establecimiento y } \\
\text { mantenimiento de la planta- } \\
\text { ción forestal }\end{array}$ & 17.78 & $\begin{array}{l}\text { 2.1 Porcentaje neto de contribución del PSA } \\
\text { sobre los costos por establecimiento y man- } \\
\text { tenimiento de la plantación forestal }\end{array}$ & 17.78 \\
\hline $\begin{array}{l}\text { 3. Generación de empleo fami- } \\
\text { liar permanente o temporal }\end{array}$ & 22.63 & $\begin{array}{l}\text { 3.1. Número de empleos a escala familiar, } \\
\text { permanentes o temporales, a los que ha te- } \\
\text { nido acceso por el PPSA }\end{array}$ & 22.63 \\
\hline $\begin{array}{l}\text { 4. Impacto en medios de } \\
\text { producción }\end{array}$ & 18.94 & $\begin{array}{l}\text { 4.1. Mejora percibida por el beneficiario en } \\
\text { condiciones de compra de herramientas por } \\
\text { un financiamiento total o parcial de los in- } \\
\text { gresos del PSA }\end{array}$ & 18.94 \\
\hline $\begin{array}{l}\text { 5. Implicaciones legales sobre } \\
\text { la finca }\end{array}$ & 19.40 & $\begin{array}{l}\text { 5.1. Cantidad de beneficiarios que no perci- } \\
\text { ben limitaciones en la finca al estar sometida } \\
\text { bajo contrato PSA para una posible futura } \\
\text { venta, con respecto al total encuestado. }\end{array}$ & 19.40 \\
\hline
\end{tabular}

Fuente: elaboración propia

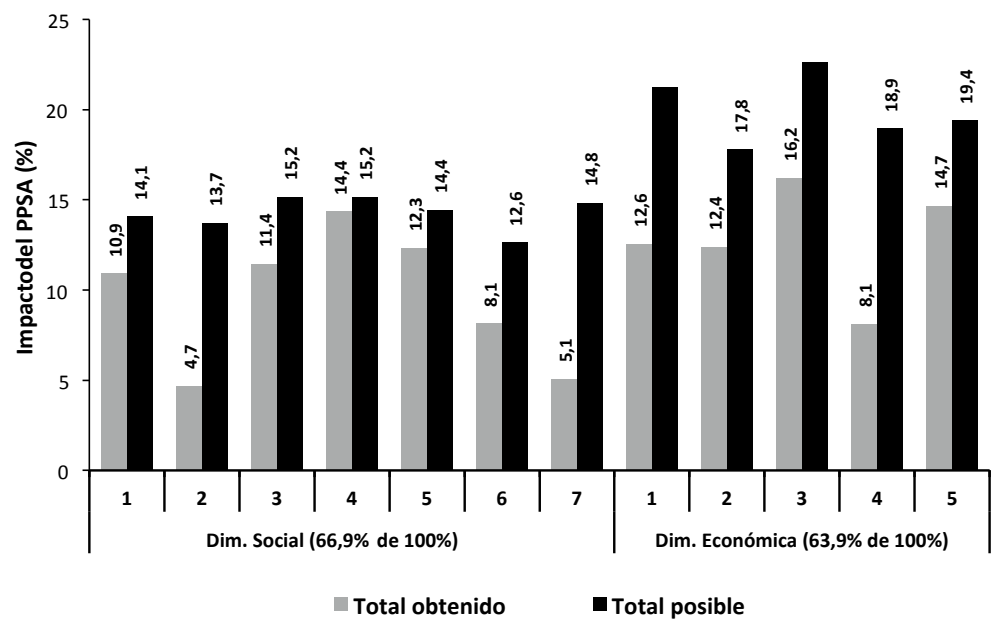

Figura 3. Puntaje obtenido y total posible para cada uno de los criterios en la evaluación general

Fuente: elaboración propia 


\section{Impacto social del Programa Pago por Servicios Ambientales en reforestadores}

En general, para esta dimensión, el impacto social correspondió a un 66.6\% (figura 3). Las áreas de mayor impacto se registran en la participación activa de los beneficiarios en sus plantaciones, así como en el nivel de satisfacción y conciencia sobre los bienes y servicios producidos (criterios 4 y 5). El $95 \%$ de los entrevistados dijo participar activamente en distintas etapas de la plantación, tales como: diseño, establecimiento y manejo (control de malezas, podas, fertilización, raleos, entre otros). Sin embargo, solamente el $51 \%$ afirmó reconocer el término de servicios ambientales, mientras que el resto lo confundió con acciones para el cuidado del medio ambiente, o lo relacionó únicamente con el nombre del programa. Según Rosa et al. (2003), además de los pagos, la capacitación es un elemento fundamental para el éxito de estas iniciativas. Aunque en nuestro estudio la mayoría de los beneficiarios ha participado en actividades de capacitación (71\%), pareciera que estas requieren mayor difusión y facilitación logística para llegar a aquellos beneficiarios en zonas más alejadas. Se evidencia una mejora técnica en el manejo de las plantaciones a partir de las capacitaciones recibidas, aunque aún se reporta una deficiencia en temas de industrialización y comercialización de productos forestales.

Adicionalmente, $76 \%$ de los beneficiarios estudiados fueron pequeños productores $(\leq 50$ ha, según priorización oficial de Fonafifo). Los pequeños productores son apoyados principalmente por medio de las OBF (Chavarría, 2013; Méndez y Salazar, 2010), las cuales facilitan la organización, algo que difícilmente sucedería sin la participación en el PPSA (Rosa et al., 2003; Camacho et al., 2002). En este estudio únicamente un $35 \%$ de los entrevistados se integró a la OBF respectiva, puesto que muchos afirmaron que ya eran miembros cuando se incorporaron al programa. Esto, a su vez, respalda el tipo de estructura de gobernanza en la que se desarrolla el PPSA, donde la gestión comunitaria, encabezada principalmente por las OBF, promueve una lógica de cooperación (Legrand et al., 2016).

\section{Impacto económico del Programa Pago por Servicios Ambientales en reforestadores}

En general, para esta dimensión, el impacto económico correspondió a un $63.9 \%$ (figura 3). El PPSA aporta, en primer lugar, para solventar una parte substancial de los costos en que incurren los productores al establecer y mantener la plantación forestal. Adicionalmente, la generación de empleo familiar y la seguridad jurídica sobre la propiedad son dos de los principales beneficios económicos percibidos. El pago por los servicios ambientales representa un $18.66 \%$ de la economía de los participantes con respecto a los demás ingresos percibidos durante el periodo de contrato. Otros estudios similares en Latinoamérica han encontrado que los pagos alcanzan alrededor del $10 \%$ del ingreso familiar (Medina, 2012; Wunder, 2005).

Aunque distintos autores señalan que el PSA puede considerarse un monto bajo y complementario, y que los pagos son insuficientes para aliviar la pobreza, sí existe una reducción de la vulnerabilidad y un impacto positivo según la condición socioeconómica de los productores (Wang et al. 2017; Suich et al., 2015; Barret et al., 2011; Camacho et al., 2002). En nuestro estudio un 24\% de los participantes logró invertir en rubros como educación, salud y gastos familiares, entre otros. Asimismo, los productores expresaron que este reconocimiento financiero les ayuda a cubrir significativamente los gastos de establecimiento y mantenimiento de la plantación, los cuales varían según condiciones físicas (geografía, estado de los caminos, disponibilidad de plántulas y riego) y humanas (disponibilidad y costo de mano de obra). Encontramos que el pago permite la generación de empleo familiar: un $46 \%$ de los beneficiarios invierte más de veinte jornales por hectárea al año y en algunos casos se generó empleo temporal en otras fincas del programa. Aunque en menor grado, los pagos además permiten la compra total o parcial de herramientas de producción, impacto modesto mencionado también por Muñoz (2012).

De manera general, se observa que los impactos sociales son mayores que los económicos, situación similar a lo encontrado en otras regiones (Molina-Murillo et al., 2014; Muñoz, 2012; Miranda 
et al., 2003). Además de lo discutido, existen causas propiamente de la gestión del programa que podrían influir en esta situación. Por ejemplo, el $39 \%$ de los productores adujo inconvenientes con la tramitología e impuntualidad en los pagos; muy lamentable, si consideramos que su principal motivación es recibir un pago para invertir en las plantaciones forestales. Factores de exclusión tales como: falta de información, procedimientos complejos, burocráticos y de alto costo, así como una débil coordinación entre sectores, una legislación fragmentada y montos que no estimulan al productor se unen a la lista de posibles causas (Borner et al., 2017; Porras, 2010; Miranda et al., 2006; Rojas, 2005; Wunder, 2005; Alpízar et al. 2003; Ortiz et al., 2003; Camacho et al., 2002). En este sentido, los beneficiarios abogan por reformas al PPSA que permitan mayor puntualidad en los pagos, disminución de requisitos y su consecuente agilización de trámites.

\section{Evaluación del impacto para todos los contratos según organizaciones de base forestal}

Al realizar el análisis por organizaciones de base forestal $(\mathrm{OBF})$, se observó que los resultados no varían significativamente para ambas dimensiones. Sus puntajes fueron 64.85 y 68.84 en la dimensión social, mientras que en la económica fueron 64.42 y 67.36, respectivamente (figura 4).

Desde el punto de vista social, es importante contar con la participación de organizaciones de pequeños productores y territorios indígenas que promueven el desarrollo de las comunidades mediante la mejora de infraestructura de uso común y el fortalecimiento de la capacidad organizativa e innovaciones tecnológicas (Molina-Murillo y Clifton, 2014), lo cual genera un impacto en la calidad de vida de los pobladores y en la manera en que son reconocidos y valorados los recursos naturales y los servicios ecosistémicos (Molina-Murillo et al., 2014; Camacho et al., 2010; Camacho et al., 2002). Según la Ley N. ${ }^{7} 7575$, la cual respalda la creación del programa, los productores que deberían verse directamente beneficiados son los pequeńos y medianos, por lo cual el rol de estas organizaciones es muy importante para el funcionamiento del PPSA, así como para incentivar la inclusión de los propietarios con menos recursos y que no podrían sufragar los costos técnicos y administrativos del programa (Porras et al., 2012; Ortiz et al., 2003). El $80 \%$ de los contratos gestionados por las OBF estudiadas son de pequeños o medianos propietarios. Las OBF se encargan también de la ubicación e inclusión de beneficiarios potenciales; el $75 \%$ dijo haberse enterado de la existencia del PPSA gracias a la promoción realizada por la $\mathrm{OBF}$, lo que además concuerda con otros estudios realizados en la zona (Alpízar et al., 2013; Chavarría, 2013; Rodríguez, 2011a; Rodríguez, 2011b; Camacho et al., 2010).

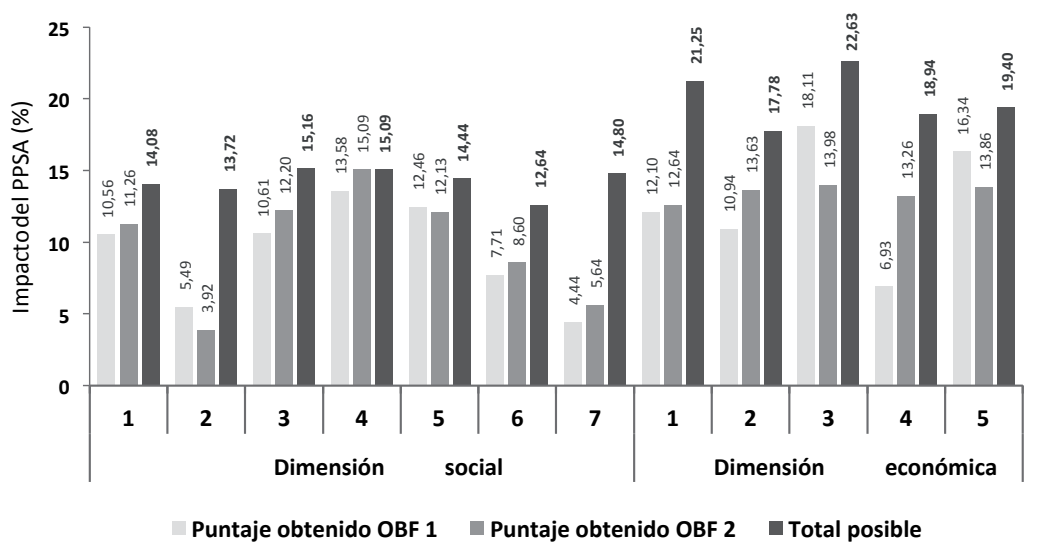

Figura 4. Comparación del puntaje de los criterios para las dos organizaciones de base forestal evaluadas

Fuente: elaboración propia 
Uno de los criterios sociales que presentó mayor diferencia fue el relacionado con el fortalecimiento de capacidades, ya que en un área, el $41 \%$ afirmó haber acudido a actividades, mientras que en la otra fue un $29 \%$; en este último caso se aludió a la dificultad para asistir por lejanía o, simplemente, por no estar enterados. A pesar de tal diferencia, este criterio fue uno de los que obtuvo mayor puntaje, lo cual demuestra que el apoyo mediante asistencia técnica y capacitaciones tales como días de campo, talleres, reuniones grupales o individuales, divulgación de material informativo, u otras, es también uno de los principales aportes de las OBF al bienestar de los productores. Otro criterio que tuvo diferencias entre las OBF fue el criterio 3, relativo al empleo familiar generado por el PPSA. Así pues, los beneficiarios que reportaron menos jornales invertidos en la actividad forestal pertenecen a la $\mathrm{OBF}$ con mayor número de residentes fuera de la provincia.

Al evaluar las OBF como intermediarias en la gestión del PPSA, el $65 \%$ de los entrevistados reportó que volverían a tramitar con ellas, mientras el resto considera que lo debería analizar o ya ha tomado la decisión de tramitarlo con regentes forestales privados. Si bien el servicio recibido fue catalogado como bueno por un $48 \%$ de las personas y regular por un $41 \%$, la falta de asistencia técnica fue la queja reincidente.

\section{Evaluación del impacto para todos los contratos según periodos}

La figura 5 evidencia que los participantes más antiguos fueron los que evaluaron de forma más positiva el impacto del PPSA en ambas dimensiones, a pesar de que en este análisis se omitió el indicador que mide el ingreso por la venta de productos, pues se asumió que aquellos productores con más tiempo ya han vendido productos intermedios o su cosecha final.

Esta tendencia a la disminución del impacto socioeconómico del PPSA a través del tiempo puede deberse a que los productores que se integraron en el primer periodo lo hicieron cuando el programa era administrado por el Sinac, con requisitos y formas de seguimiento diferentes a las de Fonafifo. También puede deberse a que los montos de los pagos no han sido actualizados de manera oportuna a través de los años; el pago en sí mismo parece que cada vez cubre en menor proporción los costos de establecimiento y mantenimiento de las plantaciones forestales. Los crecientes costos de transacción (cambios en los derechos usufructuarios o anotaciones de compraventa, donación, demandas, aviso catastral), así como los crecientes costos de mano de obra, lo cual se evidencia por un descenso en la cantidad de jornales por hectárea al año que los beneficiarios invierten en sus propias plantaciones forestales, restan competitividad a la actividad forestal.

Por último, puede deberse a un debilitamiento o cambio en la cultura agroforestal en el país, con un limitado relevo generacional. Estadísticas muestran que al 2014 solo un $27.2 \%$ de los habitantes vivía en comunidades rurales, en comparación con un $41 \%$ en el año 2000, y la población económicamente activa que labora en actividades relacionadas pasó en el mismo periodo de $18.2 \%$ a $10,5 \%$ (INEC, 2015; Facultad Latinoamericana de Ciencias Sociales [Flacso], 2002). Asimismo, el campesino costarricense tiene un promedio de edad de 54 años (INEC, 2015) y, probablemente, sus hijos no continúen con esta actividad, al considerarla poco atractiva en comparación con las opciones urbanas (Molina-Murillo, 2016).

También se encontró, lamentablemente, una relación inversa entre el año de ingreso al programa y la integración a organizaciones, la asistencia a capacitaciones y la relación con instituciones afines a la actividad agrosilvícola. Esto es desafortunado, ya que, por ejemplo, se exhibe una leve disminución en el nivel de conciencia sobre el valor de los servicios ambientales provenientes de las plantaciones (indicador 5.2), probablemente por la asistencia poco frecuente a actividades de formación y concienciación. 


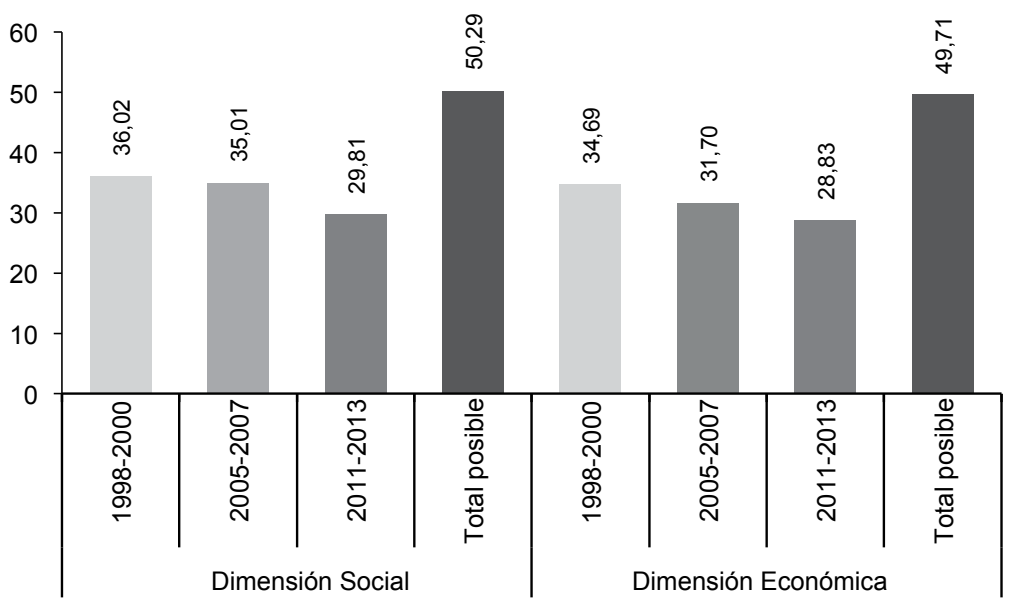

Figura 5. Comparación del puntaje de las dimensiones social y económica según periodos evaluados

Fuente: elaboración propia

\section{Conclusiones}

Con el fin de crear políticas orientadas al fortalecimiento requerido por el sector forestal, es necesario considerar el perfil de las personas reforestadoras, su percepción de la actividad y su opinión en torno a las mejoras que podrían dinamizar este sector a través de toda la cadena de valor. Incentivar un relevo generacional a través de condiciones favorables y mejoras tecnológicas en zonas rurales, mayor participación de mujeres en los procesos organizativos y de toma de decisiones, así como el fortalecimiento de las condiciones para la comercialización de la madera, por medio de la articulación de encadenamientos productivos justos, puede definir en gran parte el futuro de la reforestación. En este sentido, el PPSA ha fortalecido las capacidades locales para el desarrollo forestal de la zona, principalmente por medio de la participación activa de los beneficiarios en los diferentes procesos de sus plantaciones forestales.

Es preocupante que, a través del tiempo, pareciera haber una percepción negativa del PPSA y de sus posibles beneficios, especialmente si este es considerado un programa modelo en el ámbito tropical. A partir de la revisión documental y las múltiples entrevistas que realizamos, se recomienda partir de un enfoque de desarrollo local, fortalecer las capacidades de gestión administrativa propias y de las OBF, así como generar procesos de planificación participativos. Los impactos generados por el PPSA dependen de manera importante del entorno de los beneficiarios y otras condiciones socioeconómicas relacionadas. No obstante, para aquellos productores, principalmente pequeños y medianos, la manera en la cual opera la OBF es fundamental porque influye en la obtención de otros beneficios potenciales. Por tanto, se recomienda su fortalecimiento económico y operativo. 


\section{Agradecimientos}

A la Oficina Nacional Forestal por el apoyo financiero y logístico; a los expertos que participaron en el proceso de consulta; a la Ph. D. Miriam Miranda Quirós y al Lic. Axel Chavarría Ulate por sus aportes a la investigación; a las personas que nos facilitaron el transporte; a los funcionarios del $\mathrm{CACH}$ y $\mathrm{CACN}$, quienes muy amablemente nos brindaron la información requerida, y, principalmente, un agradecimiento a todos los beneficiarios que nos atendieron y colaboraron con su tiempo.

\section{Referencias}

Alpízar, F., Robalino, J., Sandoval, C. y Villalobos, L. (2013). Consultoría: evaluación de los impactos socioeconómicos del Programa de Pago por Servicios Ambientales. San José: s. e.

Arias, G. (2004). Análisis del impacto económico y social de las plantaciones forestales en Costa Rica. San José: s. e.

Arriagada, R. A., Ferraro, P. J., Sills, E. O., Pattanayak, S. K. y Cordero-Sancho, S. (2010). Do payments for environmental services affect forest cover? A farm-level evaluation from Costa Rica. Land Economics, $88(2), 382-399$.

Arriagada, R. A., Sills, E. O., Ferraro, P. J. y Pattanayak, S. K. (2015). Do payments pay off? Evidence from participation in Costa Rica's PES Program. PLOS ONE, 10(7), e0131544. doi:10.1371/journal. pone. 0131544

Barton, D., Faith, D., Rusch, G., Acevedo, M. y Castro, M. (2009). Environmental service payments: Evaluating biodiversity conservation trade-offs and cost-efficiency in the Osa Conservation Area, Costa Rica. Journal of Environmental Management, (90), 901-911.

Barrett, C. B., Travis, A. J. y Dasgupta, P. (2011). On biodiversity conservation and poverty traps. Proceedings of the National Academy of Sciences of the United States of America, (108), 13907-13912.

Börner, J., Baylis, K., Corbera, E., Ezzine-de-Blas, D., Honey-Rosés, J., Persson, U. M. y Wunder, S. (2017). The effectiveness of payments for environmental services. World development. doi:10.1016/j. worlddev.2017.03.020

Camacho, A., Reyes, V. y Segura, O. (2002). Gestión local y participación en torno al Pago por Servicios Ambientales: estudios de caso en Costa Rica. San Salvador: Prisma.

Camacho, A., Segura-Bonilla, O., Reyes-Gätjens, V. y Aguilar-Schramm, A. (2000). Pago por servicios ambientales punto focal: Costa Rica informe final. San José: Proyecto Prisma-Ford.

Camacho, A. y Solano, V. (2010). Los servicios ambientales en Costa Rica. San José: Instituto Interamericano de Cooperación para la Agricultura.

Chavarría, A. (2014). Las organizaciones forestales y su aporte al Programa de Pago por Servicios Ambientales (PPSA) en Costa Rica. Heredia, Costa Rica: Oficina Nacional Forestal.

Contraloría General de la República (CGR, 2011). Informe acerca de los efectos del programa pago por servicios ambientales (PSA) implementado por el Estado Costarricense. Informe Nro. DFOE-AE-08-2011. San José: autor.

Daw, T. M., Hicks, C. C., Brown, K., Chaigneau, T., Januchowski-Hartley, F. A., Cheung, W. W. L. y McClanahan, T. R. (2016). Elasticity in ecosystem services: exploring the variable relationship between ecosystems and human well-being. Ecology \& Society, 21(2), 209-221. doi:10.5751/ES-08173-210211

Engel, S., Pagiola, S. y Wunder, S. (2008). Payments for environmental services in Costa Rica. Ecological Economics, 65(4), 712-724. doi:10.1016/j.ecolecon.2007.07.033 
Facultad Latinoamericana de Ciencias Sociales (Flacso, 2002). Centroamérica en cifras. San José: Universidad de Costa Rica.

Ferraro, P. J. y Hanauer, M. M. (2014). Advances in measuring the environmental and social impacts of environmental programs. Annual Review of Environment and Resources, 39(1), 495-517. doi:10.1146/ annurev-environ-101813-013230

Fondo Internacional de Desarrollo Agrícola (FIDA, 2003). Plan detallado para un sistema de gestión de los resultados aplicable a los programas respaldados por el FIDA en los paises. Roma: autor.

Fondo Nacional Financiamiento Forestal (Fonafifo, 2015). Rendición de cuentas 2014. Recuperado de: http:// www.fonafifo.go.cr/quienesomos/rendicion/RC_Adm_Fonafifo2014.pdf

Garay, M., Locatelli, B. y Louman, B. (2004). Socio-economics impact of the payments of environmental services and the voluntary forest certification as mechanisms that promote the sustainability of forest management in Costa Rica [Impacto socioeconómico del pago por servicios ambientales y la certificación forestal voluntaria en la sostenibilidad del manejo forestal en Costa Rica]. Recursos Naturales y Ambiente (CATIE), (46-47), 99-108.

Huber-Stearns, H. R., Goldstein, J. H. y Duke, E. A. (2013). Intermediary roles and payments for ecosystem services: A typology and program feasibility application in Panama. Ecosystem Services, (6), 104-116. doi:10.1016/j.ecoser.2013.09.006

Instituto Costarricense de Estudios Sociales (ICES, 2001). Informe final del Plan de fortalecimiento de la estructura orgánica administrativa de la Municipalidad de Hojancha. Hojancha, Costa Rica: autor.

Instituto Español de Comercio Exterior (ICEX, 2012). Costa Rica: estructura económica. Oficina Económica y Comercial de la Embajada de España en Panamá. San José: autor.

Instituto Nacional de Estadísticas y Censos (INEC, 2011). X Censo Nacional de Población. San José, Costa Rica: autor.

Instituto Nacional de Estadísticas y Censos (INEC, 2015). VI Censo Nacional Agropecuario. San José, Costa Rica: autor.

Legrand, T., Le Coq, J. F. y Froger, G. (2016). Concepción de justicia y gobernanza del programa de Pago por Servicios Ambientales costarricense. Hacia un mejor entendimiento de su tipo de arreglo institucional. Revista Iberoamericana de Economía Ecológica, (26), 207-222.

León, M. (2011). La desigualdad de género en la propiedad de la tierra en América Latina. En C. Verschuur (Dir.). Du grain à moudre. Genre, développement rural et alimentation (pp. 189-207). s. 1.: s. e.

Madrigal, P., Solís, V. y Ayales, I. (2012). La experiencia forestal de Hojancha: más de 35 años de restauración forestal, desarrollo territorial y fortalecimiento social. San José, Costa Rica: Centro Agronómico Tropical de Investigación y Enseńanza, Departamento de Recursos Naturales y Ambiente. (CATIE).

Martínez, C. (2006). El método de estudio de caso: Estrategia metodológica de la investigación científica. Pensamiento y Gestión, (20), 165-193.

McGinley, K. y Finegan, B. (2001). Criterios e indicadores para evaluar la sostenibilidad ecológica: un conjunto integrado para bosques manejados en Costa Rica. Revista Forestal Centroamericana, (34), 23-27.

Medina, E. M. (2012). Impacto del programa de incentivos forestales en la ecorregión Lachuá, Guatemala. Turrialba, Costa Rica: Centro Agronómico Tropical de Investigación y Enseñanza.

Méndez, J. y Salazar, G. (2010). Las organizaciones forestales y su aporte al Programa de Pago por Servicios 
Ambientales en Costa Rica. Heredia, Costa Rica: Oficina Nacional Forestal.

Ministerio de Ambiente y Energía, Costa Rica (Minae, 2015). Decreto N.³9083. La Gaceta. Diario Oficial, Costa Rica.

Ministerio de Ambiente, Energía y Telecomunicaciones, Costa Rica. (Minaet, 2009). Manual de procedimientos para el pago de servicios ambientales. La Gaceta, Diario Oficial, Costa Rica.

Miranda, M., Dieperink, C. y Glasbergen, P. (2006). Costa Rican environmental service payments: The use of a financial instrument in participatory forest management. Environmental Management, 38(4), 562-571.

Miranda, M., Porras, I. T. y Moreno, M. L. (2003). The social impacts of payments for environmental services in Costa Rica: a quantitative field survey and analysis of the Virilla watershed. Londres: IIED.

Molina-Murillo, S. A. (2016). Desarrollo verde e inclusivo en respuesta al cambio climático. Ambientico, (258), 24-29. Recuperado de http://www.ambientico.una.ac.cr/pdfs/art/ambientico/A4.pdf

Molina Murillo, S. A. y Clifton, J. T. (2015). Perceptions of institutions and development in rural Costa Rica. Journal of Rural and Community Development, 9(4), 80-101. Recuperado de http://journals.brandonu. ca/jrcd/article/view/898

Molina Murillo, S. A., Pérez Castillo, J. P. y Herrera Ugalde, M. E. (2014). Assessment of environmental payments on indigenous territories: The case of Cabecar-Talamanca, Costa Rica. Ecosystem Services, (8), 35-43. doi:10.1016/j.ecoser.2014.02.003

Morales, S. (2016, 7 de febrero). Producción basada en servicios agrega nuevos riesgos a Costa Rica. El Financiero. Recuperado de http://www.elfinancierocr.com/finanzas/Produccion-PIB-nuevo_PIB-cambio_de_ano_ base-servicios-riesgos_0_897510246.html

Morán, M., Arce, J. J., Louman, B. y Centro Agronómico Tropical de Investigación y Enseñanza. (2006). Uso de principios, criterios e indicadores para monitorear y evaluar las acciones y efectos de politicas en el manejo de los recursos naturales. Turrialba, Costa Rica: Centro Agronómico Tropical de Investigación y Enseñanza, Departamento de Recursos Naturales y Ambiente.

Muñoz, R. (2012). Evaluación de impacto socioeconómico de los beneficiarios del Programa de Servicios Ambientales (PSA) en la modalidad de reforestación en la Zona del Programa Forestal Huetar Norte. San José: Fonafifo.

Oficina Nacional Forestal (ONF, 2012). Informe de Gestión 2009-2012: Fomentando la competitividad del sector forestal. Heredia, Costa Rica: autor.

Oficina Nacional Forestal (ONF, 2014). Usos y aportes de la madera en Costa Rica. Heredia, Costa Rica: autor.

Ortiz, E., Sage, L. y Borge, C. (2003). Impacto del Programa de Pago de Servicios Ambientales en Costa Rica como medio de reducción de la pobreza en los medios rurales. San José: s. e.

Pham, T. T., Campbell, B. M., Garnett, S., Aslin, H. y Hoang, M. H. (2010). Importance and impacts of intermediary boundary organizations in facilitating payment for environmental services in Vietnam. Environmental Conservation, 37(1), 64-72.

Porras, I. (2010). Fair and green?: social impacts of payments for environmental services in Costa Rica. Londres: IIED.

Porras, I., Miranda, M., Barton, D. y Chacón-Cascante, A. (2012). De Rio a Riot: lecciones de 20 años de experiencia en servicios ambientales en Costa Rica. Londres: International Institute for Environment and Development.

Robalino, J., Sandoval, C., Villalobos, L. y Alpízar, F. (2014). Local effects of payments for environmental services on poverty. San José: The Environment for Development. 
Rodríguez, J. (2011a). Análisis de la cadena de valor de los productos forestales que se comercializan en el cantón de Hojancha, Guanacaste, Costa Rica. Cartago, Costa Rica: Instituto Tecnológico de Costa Rica.

Rodríguez, E. (2011b). Experiencias de comercialización y uso de las plantaciones forestales en Hojancha, Guanacaste. Turrialba, Costa Rica: Centro Agronómico Tropical de Investigación y Enseñanza.

Rojas, V. (2005). Impactos socioeconómicos de un mecanismo financiero para plantaciones forestales: el caso del programa de pago por servicios ambientales en la zona norte de Costa Rica. Turrialba, Costa Rica: Centro Agronómico Tropical de Investigación y Enseñanza.

Rosa, H., Kandel, S. y Dimas, L. (2004). Compensación por servicios ambientales y comunidades rurales: lecciones de las Américas y temas críticos para fortalecer estrategías comunitarias. México, D. F., San Salvador, El Salvador: Secretaría de Medio Ambiente y Recursos Naturales, Instituto Nacional de Ecología, Consejo Civil Mexicano para la Silvicultura Sostenible, Prisma.

Serrano, M. (2005). Evaluación y planificación del manejo forestal sostenible en escala de paisaje en Hojancha, Costa Rica. Turrialba, Costa Rica: Centro Agronómico Tropical de Investigación y Enseńanza.

Sierra, R. y Russman, E. (2006). On the efficiency of environmental service payments: A forest conservation assessment in the Osa Peninsula, Costa Rica. Ecological Economics, (59), 131-141.

Smith, S., Rowcroft, P., Everard, M., Couldrick, L., Reed, M., Rogers, H., Quick, T., Eves, C. y White, C. (2013). Payments for ecosystem services: a best practice guide. Londres: Defra.

Suich, H., Howe, C. y Mace, G. (2015). Ecosystem services and poverty alleviation: A review of the empirical links. Ecosystem Services, (12), 137-147. doi:https://doi.org/10.1016/j.ecoser.2015.02.005

Valenciano, J., Zarate, D., Moreno, M. y Salas, F. (2013). Propuesta de metodología de evaluación para medir los efectos ambientales, económicos y sociales del Programa de Pago por Servicios Ambientales en Costa Rica. Heredia, Costa Rica: Centro Internacional de Política Económica para el Desarrollo Sostenible.

Wang, P., Poe, G. L. y Wolf, S. A. (2017). Payments for ecosystem services and wealth distribution. Ecological Economics, (132), 63-68. doi:10.1016/j.ecolecon.2016.10.009

Wunder, S. (2005). Pagos por servicios ambientales: Principios básicos esenciales. Jakarta, Indonesia: Center for International Forestry Research (CIFOR).

Wunder, S. (2015). Revisiting the concept of payments for environmental services. Ecological Economics, (117), 234-243. doi:10.1016/j.ecolecon.2014.08.016

Zbinden, S. y Lee, D. R. (2005). Paying for environmental services: An analysis of participation in Costa Rica's PSA Program. World Development, 33(2), 255-272. 